\title{
Propiedades Psicométricas de la Escala de Pensamientos de Enojo para Adolescentes
}

\section{Psychometric Properties of the Angry Thoughts Scale for Adolescents}

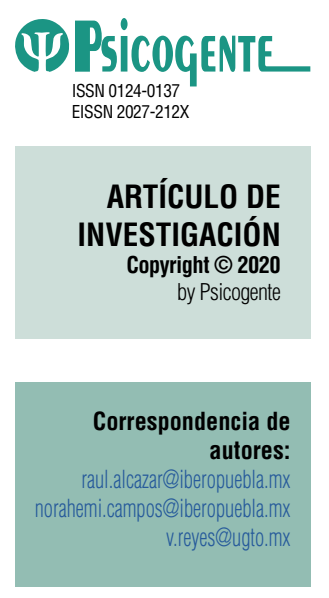

Recibido: 06/03/19 Aceptado: $13 / 08 / 19$ Publicado: 01/01/20
Raúl J. Alcázar-Olán (iD - Nora Hemi Campos Rivera (iD Universidad Iberoamericana Puebla, Puebla, México Verónica Reyes Pérez iD Universidad de Guanajuato, León, México

Resumen

Objetivo: Validar la Escala de Pensamientos de Enojo para Adolescentes, la cual incluye tres factores: venganza (4 ítems, por ej., "Haré sufrir a la otra persona"), pensamientos de insulto o peyorativos (5 ítems, por ej., "Es un estúpido") y pensamientos de control (10 ítems, por ej., "Tendré paciencia").

Método: La muestra fue de 522 adolescentes mexicanos de escuela pública ( $M$ de edad =15,92, DE =0,90); 213 hombres y 309 mujeres seleccionados de forma no aleatoria. Los instrumentos fueron: la Escala de Pensamientos de Enojo para Adolescentes, el Inventario Multicultural de Expresión de la Cólera y la Hostilidad (ML-STAXI) para Adolescentes Mexicanos y la Escala de Expresión del Enojo con Agresión Física. El diseño del estudio fue instrumental.

Resultados: Se encontró, por medio de análisis factorial confirmatorio que el modelo de tres factores tuvo buen ajuste $(\mathrm{CFI}=.912$, SRMR $=.046$, RMSEA $=.076)$. La confiabilidad (Alfa de Cronbach) fue aceptable: $.78, .86$ y .92 , respectivamente. La validez de constructo fue satisfactoria, dado que la escala fue útil para diferenciar a personas de alto y bajo puntaje en variables de enojo, control del enojo, expresión del enojo y agresión física hacia las personas y hacia los objetos. Hubo leve diferencia por sexo en una de las tres escalas, donde las mujeres tuvieron más pensamientos peyorativos que los hombres.

Conclusión: La Escala de Pensamientos de Enojo para Adolescentes es una opción confiable y con validez de constructo. Representa un punto de partida para la investigación, evaluación y tratamiento de adolescentes con problemas en el control del enojo.

Palabras clave: cogniciones de enojo; estudios psicométricos; manejo de ira; terapia cognitiva; validez.

Abstract

Objective: The goal of the study was to validate an Angry Thoughts Scale for Adolescents, which has three factors: revenge ( 4 items, e.g., "I will make the other person suffer"), insulting or pejorative thoughts (5 items, e.g., "S/he is stupid"), and thoughts of control (10 items, e.g., "I will be patient").

Method: The sample had 522 adolescents ( $M$ age $=15.92, S D=0.90) ; 213$ men, and 309 women from a public school; they were recruited through intentional non-random sampling. The participants answered three instruments: The Angry Thoughts Scale for Adolescents, The Multicultural Inventory of Anger and Hostility (ML-STAXI) for Mexican Adolescents, and the Scale of Anger Expression with Physical Aggression. The design of the study is instrumental.

Results: It was found, through confirmatory factor analysis and modification indexes, that the 3-factor model showed good fit $(\mathrm{CFI}=.912, \mathrm{SRMR}=.046, \mathrm{RMSEA}=.076)$. The reliability (Cronbach's Alpha) was acceptable: $.78, .86$, and .92 , respectively. Construct validity was satisfactory, since the scale was useful to distinguish between individuals with high and low scores in variables of anger, anger control, anger expression, and physical aggression toward people and toward objects. A gender difference was found in one of the three scales; women had more pejorative thoughts than men.

Conclusion: The Angry Thoughts Scale for Adolescents is a reliable option with construct validity. It represents a starting point for research, assessment, and treatment of adolescents with anger problems.

Key words: angry cognitions; psychometric studies; anger management; cognitive therapy; validity

Cómo citar este artículo (APA):

Alcázar-Olán, R.; Campos Rivera, N. H. \& Reyes Pérez, V. (2020). Propiedades Psicométricas de la Escala de Pensamientos de Enojo para Adolescentes.

Psicogente 23(43), 1-20. https://doi.org/10.17081/psico.22.42.3264 


\section{INTRODUCCIÓN}

El enojo es una emoción común y universal que consiste en sentimientos de irritación que van desde la leve molestia hasta la más intensa furia (Spielberger, 1999; Spielberger \& Reheiser, 2009). Esta emoción surge generalmente cuando las personas: evalúan las situaciones como injustas (por ej. considerar que "no se vale"), atribuyen daño intencionado a los demás (por ej. "lo hizo a propósito" o "esto ya es personal") y juzgan de forma peyorativa (por ej. "es un idiota" o "imbécil", entre otros). Como es de esperarse, los enojos intensos y frecuentes se asocian con resultados negativos, como peleas físicas o verbales, daño a las relaciones, destrucción de propiedades, autolesiones, consumo de alcohol, emociones negativas (vergüenza, culpa y tristeza) y consecuencias legales en el trabajo o la escuela (Cassiello-Robbins \& Barlow, 2016; Dahlen \& Martin, 2006; Deffenbacher, Oetting, Lynch \& Morris, 1996; Fernandez \& Johnson, 2016; Takebe, Takahashi \& Sato, 2016).

La presente investigación se enfoca en los adolescentes y los pensamientos que tienen cuando se enojan. Comparados con personas en otras etapas de la vida, los adolescentes presentan mayores niveles de irritabilidad, se involucran en mayor medida en altercados, cuentan con menor regulación del enojo y sienten esta emoción con más frecuencia (Blanchard-Fields \& Coats, 2008; Gamboa, Ortiz \& Muñoz, 2016; Phillips, Henry, Hosie \& Milne, 2006; Zimprich \& Mascherek, 2012). Aunque no siempre se presentan juntas, es frecuente que las reacciones iracundas y la violencia física estén asociadas (Brotman, Kirkanski \& Leibenluft, 2017). Su combinación puede ser letal, dado que la violencia interpersonal es la quinta causa de muerte en adolescentes a nivel mundial, de acuerdo con informes de la Organización Mundial de la Salud (WHO, 2018). En particular, la agresión es la segunda causa de muerte en personas mexicanas entre 15 y 24 años de edad (INEGI, 2016).

En congruencia con lo anterior, hasta el $20 \%$ de los adolescentes son fácilmente irascibles, esto es, presentan enojos frecuentes, intensos y duraderos (Alcázar-Olán \& Deffenbacher, 2013), lo cual se ha reflejado en comportamientos agresivos cotidianos hacia personas cercanas como sus hermanos o pares en la escuela (Alcázar-Olán, Jurado \& Reyes, 2015). Como resultado, es común que en escenarios clínicos se busque atención para adolescentes calificados como enojones o agresivos (Brotman et al., 2017).

Aunque se han estudiado diversos correlatos del enojo (Berkowitz \& HarmonJones, 2004; Potegal, Stemmler \& Spielberger, 2010), uno de los componentes fundamentales del enojo intenso es aquel de los pensamientos. El enojo 
exacerbado tiene un fuerte componente cognitivo. El aspecto evaluativo, las palabras o frases que pasan por la mente de la persona al sentir una emoción, es considerado como el "antecedente cognitivo más proximal de la emoción" (Smith, Haynes, Lazarus \& Pope, 1993, p.916). Entre mayor sea la presencia de ideas hostiles, juicios denigrantes hacia los demás, ideas de venganza, percepción de injusticia y daño intencionado, mayor será la presencia e intensidad del enojo (Wilkowski \& Robinson, 2010).

En el estudio del enojo y la agresión de los adolescentes, poco se ha investigado sobre sus pensamientos asociados a esta emoción. La carencia es lamentable, a pesar de que existe una fuerte conexión entre la intensidad del enojo y el contenido cognitivo de dicha emoción (Smith et al., 1993; Wilkowski \& Robinson, 2010). La medición de pensamientos de enojo en adolescentes es relevante para la detección y tratamiento de quienes tengan problemas en el manejo de esta emoción. Por ejemplo, es frecuente que los tratamientos para el manejo del enojo incluyan intervenciones de reestructuración cognitiva (Blake \& Hamrin, 2007). Sin embargo, se carece de instrumentos para evaluar el aspecto cognitivo de estas intervenciones en adolescentes.

La presente investigación evaluó en adolescentes mexicanos las propiedades psicométricas de una Escala de Pensamientos de Enojo, creada por Alcázar-Olán, Deffenbacher, Hernández-Guzmán y de la Chaussée (2014a). Dicha escala cuenta con las siguientes cinco bondades que la hacen apropiada para el presente trabajo.

Primero, la escala nació a partir de las situaciones que provocan mayor enojo en personas mexicanas (Alcázar-Olán et al., 2014a). En dicho estudio se identificaron pensamientos (palabras o frases) asociados al enojo, dentro del lenguaje español como es hablado en México. En otras palabras, los ítems de la escala son más cercanos a los adolescentes mexicanos (validez ecológica), a diferencia de las escalas de pensamientos de enojo construidas en países de habla inglesa (Deffenbacher, Petrilli, Lynch, Oetting \& Swaim, 2003; Snyder, Crowson, Houston, Kurylo \& Poirier, 1997; Martin \& Dahlen, 2007; Suchday, Carter, Ewart, Larkin \& Desiderato, 2004; Sukhodolsky, Golub \& Cromwell, 2001). Los ítems originales de otro país podrían no corresponder con un nuevo universo de generalización (el nuevo país donde se quiere adaptar la escala), lo cual podría disminuir la validez en la adaptación (Van de Vijver \& Poortinga, 2005). 
Segundo, la escala cuenta con validez cruzada, es decir, sus factores originales que surgieron en una muestra de estimación (muestra A), fueron replicados en una muestra distinta, llamada muestra de validación (muestra B). Los valores de confiabilidad se mantuvieron como aceptables, tanto en la muestra A (.79-.87) como en la B (.82-.87).

Tercero, la escala mide tres factores: dos asociados con exacerbación del enojo (ideas de venganza y pensamientos peyorativos/denigrantes) y uno asociado al autocontrol del enojo. Los tres factores representan un modelo plausible, con mejor ajuste que un modelo de solo dos factores: pensamientos de alto enojo (venganza + peyorativos) y pensamientos de autocontrol. Dada la fortaleza del modelo de tres factores (mejor ajuste), dicho modelo se consideró como la base para la presente investigación.

Cuarto, la escala cuenta con estabilidad temporal aceptable (Alcázar-Olán et al., 2014a), reflejada en valores test-retest de los tres factores para cuatro y nueve semanas, respectivamente: venganza $(r=.82$ y .65), pensamientos peyorativos ( $r=.71$ y .65 ) y pensamientos de autocontrol ( $r=.75$ y .67$)$.

Quinto, aunque existen diversos constructos sobre pensamientos de enojo, los tres factores válidos para México (venganza, pensamientos peyorativos y de autocontrol) también han sido identificados en la literatura internacional. Esto sugiere que dichos constructos son apropiados para medir pensamientos de enojo en México (Deffenbacher et al., 2003; Snyder et al., 1997; Martin \& Dahlen, 2007; Suchday et al., 2004; Sukhodolsky et al., 2001).

En síntesis, la Escala de Pensamientos de Enojo posee propiedades psicométricas aceptables, con tres factores sólidos que ya han sido replicados en población mexicana (validación cruzada). En consecuencia, se propone como hipótesis para la presente investigación que la estructura factorial de la escala será válida, es decir, será un modelo aceptable de tres factores para evaluar pensamientos de enojo en adolescentes mexicanos.

En cuanto al sexo, las investigaciones han encontrado pocas diferencias en términos de sentir y expresar el enojo. El estudio meta-analítico de Archer (2004) reportó que hombres y mujeres son parecidos en esta emoción. Además, cuando los estudios han hallado diferencias, éstas han explicado poca varianza. Por ejemplo, los hombres han mostrado más ideas de venganza que las mujeres, así como más ideas de autocontrol; la varianza explicada fue menor al 1,6\% (Alcázar-Olán et al., 2014a). Deffenbacher y otros (2003) 
encontraron más pensamientos de venganza y agresión física en los hombres, con varianza explicada del 11,2 al $14,7 \%$. Por el contrario, Martin y Dahlen (2007) hallaron diferencias en sólo una de las seis variables sobre cogniciones de enojo, donde los hombres presentaron más pensamientos con adjetivos denigrantes; la varianza explicada fue del 1,9\%. Este panorama sugiere pocas diferencias entre hombres y mujeres en cuanto al enojo y a los pensamientos asociados a esta emoción. A partir de estas evidencias, se propone para el presente estudio como hipótesis que no habrá diferencias por sexo y, en caso de haberlas, serán mínimas, con baja varianza explicada igual que en estudios previos.

El objetivo de esta investigación fue validar el uso de la Escala de Pensamientos de Enojo (Alcázar-Olán et al., 2014a) en adolescentes mexicanos. El presente objetivo tiene dos fundamentos. Primero, las propuestas de instrumentos deben ser sometidas a un examen de su rigurosidad científica. En particular, debe analizarse su validez, entendida como la "utilidad científica de un instrumento de medida" (Nunnally \& Bernstein, 1995, p.91). Segundo, la validación permite hacer una "prueba empírica del significado y consecuencias de la medición" (Messick, 1995, p.742), con lo cual la validación combina la búsqueda científica con el argumento racional de justificar (o anular) el uso e interpretación de los puntajes de alguna escala.

\section{MÉTODO}

\subsection{Diseño}

La presente investigación es de tipo instrumental (Ato, López \& Benavente, 2013), dado que incluye el estudio de propiedades psicométricas de un instrumento de medida psicológica.

\subsection{Participantes}

La muestra se eligió de manera no aleatoria y se formó de 522 adolescentes (213 hombres y 309 mujeres) de nivel escolar denominado preparatoria, la cual corresponde en México al ciclo de estudio de tres años para jóvenes de, aproximadamente, 15 a 18 años. El tipo de escuela fue pública. La edad promedio de los participantes fue de 15,92 (DT =0,90). El 43,1\% cursaba el primer año de preparatoria; el $36,8 \%$, segundo; y el $20,1 \%$, tercero. Los criterios de inclusión fueron estar en el rango de edad entre 15 y 18 años y estar estudiando el nivel escolar de preparatoria; el segundo criterio aseguró que los participantes supieran leer y escribir. 


\subsection{Instrumentos}

\subsubsection{Inventario Multicultural de Expresión de la Cólera y la Hostilidad (ML-STAXI) para Adolescentes Mexicanos (Alcázar-Olán et al., 2014b)}

Este inventario se basa en la versión original para medir la experiencia y expresión del enojo en adultos (Moscoso, 2000). La adaptación para adolescentes mexicanos de nivel preparatoria incluye las siguientes escalas:

La escala de Enojo-estado mide la intensidad del enojo (10 ítems, $\alpha=.84$ ) por medio de dos subescalas: Sentimientos de enojo (5 ítems, $\alpha=.74$ ), por ejemplo, "Estoy furioso" y Deseos de mostrar enojo (5 ítems, $\alpha=.85$ ), por ejemplo, "Tengo ganas de insultar a alguien". La escala de Enojo-rasgo (10 ítems, $\alpha=.81$ ) mide la tendencia a enojarse fácilmente en general y ante provocaciones específicas. Esta escala se forma de dos subescalas: Temperamento-iracundo (5 ítems, $\alpha=.79$ ), por ejemplo, "Soy muy temperamental" y Reacción de enojo (5 ítems, $\alpha=.78$ ), por ejemplo, "Me enojo cuando alguien arruina mis planes".

En cuanto a la expresión y manejo del enojo, las escalas son las siguientes: Control del enojo interno (4 ítems, $\alpha=.78$ ), por ejemplo, "Cuando me enojo, trato de relajarme"; Control del enojo externo ( 6 ítems, $\alpha=.78$ ), por ejemplo, "Cuando me enojo, controlo mi forma de reaccionar"; indicador de Reducción rápida del enojo ( 2 ítems, $\alpha=.88$ ), por ejemplo, "Trato de calmarme lo más pronto posible"; Enojoexterno (3 ítems, $\alpha=.65$ ), por ejemplo, "Muestro mi enojo a los demás"; y Enojointerno (6 ítems, .63), por ejemplo, "Suprimo mi enojo varias horas". La escala de Enojo-estado se responde con las opciones de 1 (No en absoluto) a 4 (Mucho); mayor puntaje refleja más intensidad del enojo. Las demás escalas se responden con las opciones de 1 (Casi nunca) a 4 (Casi siempre), donde mayor puntaje indica mayor frecuencia en lo que describen los ítems. La adaptación del ML-STAXI para adolescentes mexicanos indica validez factorial (Alcázar-Olán et al., 2014b) similar a la escala original (Moscoso, 2000).

\subsubsection{Escala de Pensamientos de Enojo (Alcázar-Olán et al., 2014a)}

Esta escala se creó para adultos mexicanos y se compone de tres dimensiones para medir la frecuencia en que se presentan pensamientos de enojo. Las personas responden a la instrucción: "Cuando te enojas, ¿̇con qué frecuencia tienes los siguientes pensamientos?". Luego, aparecen los ítems. La escala de Pensamientos de venganza ( 4 ítems, $\alpha=.79$ ) incluye ideas de represalia, por ejemplo, "Haré sufrir a la otra persona". La escala de Pensamientos peyorativos ( 5 ítems, $\alpha=.83$ ) se compone de adjetivos de desprecio o denigrantes, 
por ejemplo, "Es un idiota". La escala de Pensamientos de control del enojo (10 ítems, $\alpha=.87$ ) mide autoinstrucciones para el manejo del enojo, por ejemplo, "Mejor mantendré la calma". Las opciones de respuesta van de 1 (Casi nunca) a 4 (Casi siempre). La escala tiene validez de constructo y su estructura factorial se replicó en una muestra diferente.

\subsubsection{Escala de Expresión del Enojo con Agresión Física (Alcázar-Olán et al., 2015)}

Esta escala se construyó para otra investigación, la cual examinó su validez factorial. La escala midió la presencia de agresiones físicas recientes por medio de dos subescalas. La primera (6 ítems, $\alpha=.77$ ) evaluó la expresión del enojo hacia otras personas, con ítems sobre golpear a alguien, jalonear, hacer como si fuera a dar un golpe, retar a golpes, romper cosas de alguien y aventar cosas contra alguien. La segunda ( 6 ítems, $\alpha=.71$ ) midió la expresión del enojo hacia objetos o el ambiente, con ítems sobre dañar cosas propias, hacer como si fuera a romper algo, golpear la pared, azotar la puerta, aventar cosas y romper objetos. Los adolescentes respondieron a la pregunta: “¿Cuándo fue la última vez que estando enojado...?” y luego aparecieron los ítems. Las opciones de respuesta fueron desde $1=$ Nunca lo he hecho hasta 7 = Lo hice hoy. Mayor puntaje indica expresión del enojo con agresión física recientemente.

\subsection{Procedimiento}

Primero se consiguió la autorización de una escuela pública de nivel preparatorio de turno matutino. El permiso se gestionó con los directivos y ellos lo hablaron con el comité de padres de familia, el cual aceptó. Como agradecimiento, los resultados de la investigación se expusieron en esa escuela preparatoria.

Los cuestionarios se aplicaron en ausencia del profesor, en grupos de aproximadamente 25 alumnos cada uno. Las instrucciones se leyeron en voz alta, incluyendo la aclaración de confidencialidad y anonimato, así como la naturaleza voluntaria del estudio.

\subsection{Análisis de resultados}

Con respecto al objetivo de validar el uso de la Escala de Pensamientos de Enojo en adolescentes mexicanos, el análisis estadístico se realizó con el programa EQS 6.0 (Bentler, 1995), el cual permitió hacer el análisis factorial confirmatorio. Se utilizaron los métodos robustos de máxima verosimilitud del EQS (Bentler, 1995) debido a que el estimado de la Kurtosis multivariada 
normalizada fue de 35,60, lo cual indicó distribución no normal. Los resultados se interpretaron por medio del grado de ajuste del AFC, con base en los siguientes estadísticos: 1) $X^{2}$ de Satorra-Bentler (2001), donde valores no significativos indican buen ajuste, aunque su valor es muy sensible al tamaño de la muestra, de manera que un buen modelo puede resultar con pobre ajuste; 2) el índice de ajuste comparativo (CFI) y el índice de ajuste comparativo robusto (RCFI), donde valores arriba de .90 indican ajuste aceptable (Bentler, 1990); 3) el residuo estandarizado de la raíz cuadrada promedio (SRMR), donde valores debajo de .08 se consideran buenos (Hu \& Bentler, 1999, p.1) y la raíz cuadrada promedio del error de aproximación (RMSEA), donde valores debajo de .06 se consideran buenos (Hu \& Bentler, 1999, p. 27).

Los demás análisis se realizaron con el programa SPSS versión 17. Se realizó un análisis de varianza para examinar las diferencias por sexo. El resultado se interpretó con el criterio tradicional de significancia estadística $(p<.05)$ y con criterios para evaluar el tamaño del efecto o varianza explicada (Eta cuadrada: $\eta^{2}$ ). Este índice se evaluó con los criterios propuestos por Cohen (1988), donde puntajes de .01 a .04 se consideran pequeños; de .04 a .14, moderados; y mayores de .14, grandes.

Dado que el objetivo de investigación fue validar el uso de la Escala de Pensamientos de Enojo, la escala se examinó también por medio de correlaciones con otros instrumentos ya existentes (ML-STAXI y Escala de Expresión del Enojo con Agresión Física). Las correlaciones fueron interpretadas con los criterios de Cohen (1988), dado que son apropiados en el área de ciencias conductuales. En apego al autor, correlaciones con valores $r$ debajo de $.10\left(r^{2}\right.$ $=.01)$ se consideran pequeñas, de $.30\left(r^{2}=.09\right)$, moderadas y arriba de .50 $\left(r^{2}=.25\right)$, fuertes (Cohen, 1988, pp.79-80). La varianza explicada $\left(r^{2}\right)$ se tomó como indicador del grado en que la variación en una de las subescalas es atribuible a otra subescala (Cohen, 1988, pp.79-80).

Finalmente, se usó el método de contrastar grupos (Cohen \& Swerdlik, 2009) para examinar si las subescalas de Pensamientos de Enojo (grupos de alto y bajo puntaje) son útiles como variable independiente para distinguir puntajes de otras variables (variables dependientes). Las diferencias entre grupos se examinaron con análisis de varianza y el resultado se interpretó con el criterio tradicional de significancia estadística $(p<.05)$ y con los criterios arriba mencionados de Cohen (1988) para evaluar el tamaño del efecto o varianza explicada (Eta cuadrada: $\eta^{2}$ ). 


\subsection{Conflicto de interés}

Los autores declaran la ausencia de conflicto de interés en la elaboración de este trabajo.

\section{RESULTADOS}

\subsection{1. Análisis Factorial Confirmatorio}

Se examinó el modelo de tres factores, provenientes de la escala original: pensamientos de venganza, peyorativos y control del enojo (Alcázar-Olán et al., 2014a). Como resultado, la $X^{2}$ de Satorra-Bentler (2001) fue significativa $\left(X^{2}=474.32, g l=149, p=.000\right)$, aunque esto no es extraño en muestras grandes. El modelo tuvo ajuste aceptable en el CFI normal (.912) y robusto (.927), así como en el valor SRMR (.046), aunque los valores de RMSEA normal (.076; IC del 90\%: .070-.083) y robusto (.065; IC del 90\%: .058-.071) fueron marginales. Dado el ajuste aceptable en la mayoría de los índices, dentro de los criterios arriba establecidos, se decidió no rechazar este modelo de tres factores. La Tabla 1 muestra las cargas estandarizadas para cada ítem.

Tabla 1.

Análisis factorial confirmatorio de la Escala de Pensamientos de Enojo

\begin{tabular}{|c|c|c|c|c|c|}
\hline FACTOR & ÍTEM & CARGA & M & DE & ALFA AL ELIMINAR EL ÍTEM \\
\hline Pensamientos & Me voy a desquitar & .76 & 1,88 & 0,95 & .71 \\
\hline \multirow[t]{3}{*}{ de venganza } & Haré sufrir a la otra persona & .72 & 1,53 & 0,87 & .73 \\
\hline & Le daré su merecido & .69 & 1,68 & 0,90 & .74 \\
\hline & Haré algo en contra de los demás & .62 & 1,46 & 0,81 & .76 \\
\hline Pensamientos & Él(ella) es un(a) imbécil & .81 & 1,89 & 0,98 & .81 \\
\hline \multirow[t]{4}{*}{ peyorativos } & Gente estúpida & .77 & 1,99 & 1,00 & .82 \\
\hline & Es un(a) estúpido(a) & .74 & 2,11 & 1,05 & .83 \\
\hline & Él(ella) es un maldito(a) & .73 & 1,90 & 0,95 & .83 \\
\hline & Es un(a) hipócrita & .66 & 2,17 & 1,08 & .84 \\
\hline Pensamientos & Intento relajarme & .86 & 2,61 & 1,03 & .90 \\
\hline \multirow[t]{9}{*}{ de control } & Es mejor razonar que enojarse & .77 & 2,39 & 1,05 & .91 \\
\hline & Tendré paciencia & .75 & 2,52 & 1,01 & .90 \\
\hline & Mantendré el control & .74 & 2,56 & 1,01 & .91 \\
\hline & $\begin{array}{c}\text { Buscaré un lado positivo a la } \\
\text { situación }\end{array}$ & .73 & 2,49 & 1,05 & .91 \\
\hline & Pienso en resolver el problema & .70 & 2,51 & 1,03 & .91 \\
\hline & Mejor mantendré la calma & .70 & 2,51 & 1,06 & .91 \\
\hline & Intento ser paciente con los demás & .70 & 2,47 & 1,04 & .91 \\
\hline & $\begin{array}{c}\text { Haré un plan para resolver el } \\
\text { problema }\end{array}$ & .62 & 2,29 & 1,05 & .91 \\
\hline & Le busco un remedio a la situación & .61 & 2,44 & 1,03 & .91 \\
\hline
\end{tabular}




\subsection{Confiabilidad}

La confiabilidad se examinó con el Alfa de Cronbach. Los valores fueron aceptables para las escalas de pensamientos de venganza $(\alpha=.78)$, pensamientos peyorativos $(\alpha=.86)$ y pensamientos de control del enojo $(\alpha=.92)$. Al revisar los valores alfa de cada ítem, en el caso de eliminar al ítem de la escala, se encontró que todos contribuyen al alfa total y, la eliminación de algún ítem, no incrementaría la confiabilidad (Tabla 1). En consecuencia, cada ítem contribuye a la escala a la que pertenece.

\subsection{Análisis por sexo}

Se comparó a hombres y mujeres en cuanto a sus pensamientos de enojo. Como resultado, se encontró diferencia en una de las tres escalas. Las mujeres tuvieron más pensamientos peyorativos que los hombres (Tabla 2). El tamaño del efecto fue pequeño, según el criterio de Cohen (1988), con lo cual se explica poca varianza $(1,4 \%)$ al comparar ambos sexos. Hombres y mujeres parecen más similares que distintos en sus pensamientos de enojo.

Tabla 2.

Análisis por sexo en la Escala de Pensamientos de Enojo

\begin{tabular}{|c|c|c|c|c|c|c|c|c|}
\hline \multirow{2}{*}{ TIPOS DE PENSAMIENTOS } & \multicolumn{2}{|c|}{ MUESTRA TOTAL } & \multicolumn{2}{|c|}{ HOMBRES } & \multicolumn{2}{|c|}{ MUJERES } & \multirow{2}{*}{$\begin{array}{c}\text { ANOVA } F \\
(1,520)\end{array}$} & \multirow{2}{*}{$\begin{array}{l}\text { TAMAÑO DEL } \\
\text { EFECTO } \\
\left(\mathrm{H}^{2}\right)\end{array}$} \\
\hline & M & DE & M & DE & M & DE & & \\
\hline Venganza & 6,54 & 2,78 & 6,72 & 2,59 & 6,42 & 2,90 & 1,44 & .003 \\
\hline Peyorativos & 10,06 & 4,06 & 9,48 & 3,84 & 10,46 & 4,17 & $7,36^{*}$ & .014 \\
\hline Control del enojo & 24,78 & 7,93 & 25,35 & 7,78 & 24,39 & 8,03 & 1,87 & .004 \\
\hline
\end{tabular}

${ }^{*} p<.01$

\subsection{Correlaciones entre Escalas y otras Mediciones}

Las escalas de venganza y pensamientos peyorativos tuvieron correlación de .57 , la cual se considera fuerte, según el criterio de Cohen (1988, p. 80). Este valor es esperado con base en que se trata de tipos de pensamientos de exacerbación del enojo, ambos pertenecientes a la misma escala que se valida en el presente estudio.

Adicionalmente, las escalas de venganza y pensamientos peyorativos tuvieron correlación de -.26 y -.16, respectivamente, con la escala de pensamientos de autocontrol del enojo. Estos valores se consideran correlaciones bajas de acuerdo con el criterio de Cohen (1988, p.79-80), por debajo de los valores de correlación moderada de .30 (Cohen, 1988, p.80). Estas correlaciones (-.26 y -.16) son esperadas, debido a que, al igual que en otros estudios (Alcázar-Olán et al., 2014a, p.383; Deffenbacher et al., 2003, p.394), los pensamientos de exacerbación del enojo (venganza y peyorativos) y los pensamientos de 
autocontrol son en gran medida independientes, lo cual se refleja en la baja varianza explicada, con $r^{2}=.067$ entre venganza y autocontrol, y $r^{2}=.025$ entre peyorativos y autocontrol. Esto es, los pensamientos de exacerbación del enojo (venganza y peyorativos) y pensamientos de autocontrol no forman parte de un mismo continuum.

También se analizó, con los criterios de Cohen (1988, pp.79-81) el grado en que la escala del presente estudio se correlaciona con medidas ya existentes, en particular con el inventario de enojo (ML-STAXI) y la escala de agresión física reciente (Tabla 3). Se encontró que las escalas de venganza y pensamientos peyorativos tuvieron correlaciones positivas de bajas a moderadas con escalas de deseos de mostrar enojo, sentir enojo, enojo-rasgo, expresión del enojo y con agresión física hacia las personas y hacia los objetos. Por su parte, la escala de pensamientos de control del enojo tuvo correlaciones positivas fuertes con escalas de control del enojo interno y externo, así como con la reducción rápida del enojo. En síntesis, la Escala de Pensamientos de Enojo tuvo correlaciones de bajas a fuertes con otros instrumentos de enojo, lo cual aporta un apoyo inicial de validez convergente.

Tabla 3.

Correlaciones entre las escalas de Pensamientos de Enojo y otras escalas

\begin{tabular}{|c|c|c|c|c|}
\hline \multirow{2}{*}{ ESCALAS } & \multirow{2}{*}{ SUBESCALAS } & \multicolumn{3}{|c|}{ TIPOS DE PENSAMIENTOS DE ENOJO } \\
\hline & & VENGANZA & PEYORATIVOS & AUTOCONTROL \\
\hline Pensamientos & Peyorativos & .57 & & \\
\hline de enojo & Autocontrol & -.26 & -.16 & \\
\hline \multirow[t]{10}{*}{ ML-STAXI ${ }^{1}$} & Deseos de mostrar enojo & .34 & .26 & -.13 \\
\hline & Sentimientos de enojo & .20 & .25 & -.07 \\
\hline & Enojo-rasgo & .39 & .45 & -.18 \\
\hline & Temperamento iracundo & .32 & .35 & -.24 \\
\hline & Reacción de enojo & .35 & .43 & -.07 \\
\hline & Control de enojo interno & -.07 & -.01 & .57 \\
\hline & Control de enojo externo & -.15 & -.13 & .59 \\
\hline & Enojo externo & .35 & .40 & -.18 \\
\hline & Enojo interno & .36 & .36 & -.04 \\
\hline & Reducción rápida de enojo & -.11 & -.05 & .57 \\
\hline Agresión física & Hacia personas & .43 & .31 & -.24 \\
\hline reciente & Hacia objetos & .33 & .36 & -.19 \\
\hline
\end{tabular}

Nota. 1ML-STAXI = Inventario Multicultural Latinoamericano de Expresión de la Cólera y la Hostilidad; $r>.10, p<.01 ; r\rangle$ $.15, p<.001$.

\subsection{Validez de Constructo}

Se usó el método de "contrastar grupos" (Cohen \& Swerdlik, 2009) para evaluar la validez de constructo. El método supone que, si la escala es una medida válida de un constructo particular, entonces los puntajes de distintos grupos tendrían que diferir. Para ello, se evaluó si las subescalas de Pensamientos 
de Enojo logran distinguir con respecto a otras pruebas existentes. Estas pruebas fueron el ML-STAXI y la Escala de Expresión del Enojo con Agresión Física. En particular, se examinó si la Escala de Pensamientos de Enojo logra diferenciar entre un grupo normativo y uno con puntajes sugerentes de problemas clínicos.

Para ello, se identificaron los cuartiles de la Escala de Pensamientos de Enojo en Adolescentes. Los cuartiles son un punto de corte frecuente para detectar a personas con problemas de enojo (DiGiuseppe \& Tafrate, 2003), lo cual indica altos puntajes en sentir y expresar esta emoción.

Con base en los cuartiles $(Q)$ de la Escala de Venganza se formaron dos grupos: normativo de venganza (Q1-Q3; puntajes $4-7 ; n=378$ ) y alto puntaje de venganza (cuartil alto o Q4; puntajes $8-16 ; n=144$ ). Ambos grupos fueron la variable independiente, mientras que las variables dependientes se representaron por las mediciones del ML-STAXI y la Escala de Expresión del Enojo con Agresión Física. Como resultado, la Escala de Venganza discriminó satisfactoriamente en todas las variables dependientes (Tabla 4). Las personas con alto puntaje en venganza tuvieron más deseos de expresar el enojo y mayores conductas agresivas, a diferencia de las personas con puntaje normativo en venganza (Tabla 4). Por lo tanto, la escala de venganza tuvo validez de constructo porque distinguió correctamente a ambos grupos. Esta lógica comparativa también se usó en los siguientes análisis, con la Escala de Pensamientos Peyorativos y de Control del Enojo.

Tabla 4.

La Escala de Venganza y el contraste entre grupo normativo y alto puntaje

\begin{tabular}{|c|c|c|c|c|c|c|}
\hline \multirow[t]{2}{*}{$\begin{array}{c}\text { ESCALAS DE ENOJO Y } \\
\text { AGRESIÓN FÍSICA }\end{array}$} & \multicolumn{2}{|c|}{$\begin{array}{c}\text { GRUPO NORMATIVO } \\
\text { EN PUNTAJE DE VENGANZA }\end{array}$} & \multicolumn{2}{|c|}{$\begin{array}{c}\text { GRUPO CON ALTO } \\
\text { PUNTAJE } \\
\text { DE VENGANZA }\end{array}$} & \multirow[t]{2}{*}{$\begin{array}{l}\text { ANOVA } F \\
(1,520)\end{array}$} & \multirow[t]{2}{*}{$\begin{array}{c}\text { TAMAÑO } \\
\text { DEL EFECTO } \\
\left(\mathrm{H}^{2}\right)\end{array}$} \\
\hline & $\mathbf{M}$ & DE & M & DE & & \\
\hline Deseos de mostrar enojo & 5,48 & 1,06 & 6,88 & 2,96 & $62,47^{* * *}$ & .107 \\
\hline Sentimientos de enojo & 6,07 & 1,50 & 6,85 & 2,24 & $20,99 * * *$ & .039 \\
\hline Enojo-rasgo & 18,30 & 5,43 & 23,18 & 6,57 & $73,93 * * *$ & .124 \\
\hline Temperamento iracundo & 8,56 & 3,04 & 11,02 & 4,02 & $56,66^{* * *}$ & .098 \\
\hline Reacción de enojo & 9,74 & 3,38 & 12,16 & 3,68 & $50,61^{* * *}$ & .098 \\
\hline Control de enojo interno & 11,14 & 3,29 & 10,08 & 3,56 & $10,43^{* *}$ & .020 \\
\hline Control de enojo externo & 14,98 & 4,25 & 13,69 & 3,84 & $10,18 * *$ & .019 \\
\hline Enojo externo & 5,60 & 1,99 & 7,20 & 2,58 & $56,72 * * *$ & .098 \\
\hline Enojo interno & 10,45 & 3,19 & 12,74 & 3,74 & $48,54^{* * *}$ & .085 \\
\hline Reducción rápida de enojo & 5,61 & 1,84 & 4,99 & 1,90 & $11,61 * *$ & .022 \\
\hline Agresión hacia personas & 12,79 & 6,49 & 19,53 & 8,42 & $94,61^{* * *}$ & .154 \\
\hline Agresión hacia objetos & 14,41 & 6,40 & 18,81 & 7,44 & $44,93 * * *$ & .080 \\
\hline
\end{tabular}

${ }^{* *} p<.01,{ }^{* * *} p<.001$ 
Los puntajes de la Escala de Pensamientos Peyorativos se dividieron en dos grupos: normativo (Q1-Q3; puntajes $5-12 ; n=392$ ) y alto puntaje en pensamientos peyorativos (cuartil alto o Q4; puntaje 13-20; $n=130$ ). Como resultado, la escala discriminó satisfactoriamente en 10 de las 12 escalas criterio (Tabla 5). Esto es, diferenció a personas de alto y bajo puntaje en 10 variables asociadas al enojo. De forma no sorpresiva, no distinguió altos y bajos puntajes sobre tratar de controlar el enojo por medio de respirar profundo y relajarse rápidamente.

Tabla 5.

Escala de Pensamientos Peyorativos y el contraste entre grupos

\begin{tabular}{|c|c|c|c|c|c|c|}
\hline \multirow[t]{2}{*}{$\begin{array}{l}\text { ESCALAS DE ENOJO Y } \\
\text { AGRESIÓN FÍSICA }\end{array}$} & \multicolumn{2}{|c|}{$\begin{array}{l}\text { GRUPO NORMATIVO } \\
\text { EN PENSAMIENTOS } \\
\text { PEYORATIVOS }\end{array}$} & \multicolumn{2}{|c|}{$\begin{array}{l}\text { GRUPO DE PUNTAJE } \\
\text { PEYORATIVO } \\
\text { ALTO }\end{array}$} & \multirow[t]{2}{*}{$\begin{array}{l}\text { ANOVA } F \\
(1,520)\end{array}$} & \multirow{2}{*}{$\begin{array}{c}\text { TAMAÑO } \\
\text { DEL EFECTO } \\
\left(\mathrm{H}^{2}\right)\end{array}$} \\
\hline & M & $\mathrm{DE}$ & M & $\mathrm{DE}$ & & \\
\hline Deseos de mostrar enojo & 5,62 & 1,45 & 6,60 & 2,73 & $27,14^{* * *}$ & .050 \\
\hline Sentimientos de enojo & 6,08 & 1,64 & 6,88 & 1,99 & $20,76^{* * *}$ & .038 \\
\hline Enojo-rasgo & 18,31 & 5,46 & 23,67 & 6,53 & $84,93 * * *$ & .140 \\
\hline Temperamento iracundo & 8,67 & 3,13 & 10,93 & 4,04 & $43,45^{* * *}$ & .077 \\
\hline Reacción de enojo & 9,64 & 3,24 & 12,74 & 3,74 & $82,36 * * *$ & .137 \\
\hline Control de enojo interno & 10,84 & 3,36 & 10,88 & 3,53 & 0,012 & .000 \\
\hline Control de enojo externo & 14,88 & 4,29 & 13,85 & 3,75 & $5,95^{*}$ & .011 \\
\hline Enojo externo & 5,53 & 1,94 & 7,60 & 2,52 & $95,11 * * *$ & .155 \\
\hline Enojo interno & 10,46 & 3,19 & 12,94 & 3,72 & $53,69 * * *$ & .094 \\
\hline Reducción rápida de enojo & 5,47 & 1,87 & 5,32 & 1,88 & 0,61 & .001 \\
\hline Agresión hacia personas & 13,51 & 7,21 & 18,08 & 8,05 & $37,08^{* * *}$ & .067 \\
\hline Agresión hacia objetos & 14,53 & 6,66 & 18,92 & 6,93 & $41,63 * * *$ & .074 \\
\hline
\end{tabular}

${ }^{*} p<.05, * * * p<.001$

Con la Escala de Pensamientos de Control del Enojo se formaron dos grupos: poco control en pensamientos de enojo (cuartil bajo o Q1; puntajes 10-19; $n=$ 147 ) y puntaje normativo (Q2-Q4; puntajes $20-40 ; n=375)$. Como resultado, la escala discriminó satisfactoriamente en nueve de las 12 escalas criterio (Tabla 6). Como era de esperarse, dado que la escala midió control, discriminó en cuanto reducción rápida del enojo y el control del enojo interno y externo; también, en deseos de mostrar enojo, temperamentoiracundo y agresión física hacia otras personas y hacia objetos. No discriminó en enojointerno, enojoexterno, reacción de enojo, ni en sentimientos de enojo. 
Tabla 6.

Escala de Pensamientos de Control del Enojo y el contraste entre grupos

\begin{tabular}{|c|c|c|c|c|c|c|}
\hline \multirow[t]{2}{*}{$\begin{array}{l}\text { ESCALAS DE ENOJO Y } \\
\text { AGRESIÓN FÍSICA }\end{array}$} & \multicolumn{2}{|c|}{$\begin{array}{l}\text { GRUPO DE BAJO } \\
\text { CONTROL } \\
\text { DEL ENOJO }\end{array}$} & \multicolumn{2}{|c|}{$\begin{array}{l}\text { GRUPO DE PUNTAJE NOR- } \\
\text { MATIVO EN CONTROL DEL } \\
\text { ENOJO }\end{array}$} & \multirow[t]{2}{*}{$\begin{array}{c}\text { ANOVA } F \\
(1,520)\end{array}$} & \multirow{2}{*}{$\begin{array}{c}\text { TAMAÑO } \\
\text { DEL EFECTO } \\
\left(\mathrm{H}^{2}\right)\end{array}$} \\
\hline & $M$ & $\mathrm{DE}$ & $M$ & $\mathrm{DE}$ & & \\
\hline Deseos de mostrar enojo & 6,26 & 2,52 & 5,71 & 1,56 & $8,87^{* *}$ & .017 \\
\hline Sentimientos de enojo & 6,30 & 1,61 & 6,27 & 1,83 & 0,02 & .000 \\
\hline Enojo-rasgo & 20,56 & 7,23 & 19,29 & 5,69 & $4,54^{*}$ & .009 \\
\hline Temperamento iracundo & 10,11 & 3,94 & 8,89 & 3,27 & $12,89 * * *$ & .024 \\
\hline Reacción de enojo & 10,46 & 4,07 & 10,39 & 3,44 & 0,03 & .000 \\
\hline Control de enojo interno & 8,33 & 3,10 & 11,83 & 2,98 & $142,25^{* * *}$ & .215 \\
\hline Control de enojo externo & 11,60 & 3,23 & 15,81 & 3,91 & $134,46^{* * *}$ & .205 \\
\hline Enojo externo & 6,44 & 2,55 & 5,89 & 2,15 & $6,34^{*}$ & .012 \\
\hline Enojo interno & 11,03 & 3,79 & 11,10 & 3,38 & 0,03 & .000 \\
\hline Reducción rápida de enojo & 4,14 & 1,61 & 5,94 & 1,72 & $119,15^{* * *}$ & .186 \\
\hline Agresión hacia personas & 16,16 & 8,36 & 14,06 & 7,32 & $7,99 * *$ & .015 \\
\hline Agresión hacia objetos & 16,59 & 7,89 & 15,25 & 6,57 & $3,89 *$ & .007 \\
\hline
\end{tabular}

${ }^{*} p<.05,{ }^{* *} p<.01,{ }^{* * *} p<.001$.

En síntesis, la Escala de Pensamientos de Enojo fue útil, con base en sus altos y bajos puntajes, para encontrar diferencias en diversas variables de enojo y agresión.

\section{DISCUSIÓN}

La estructura de tres factores (pensamientos de venganza, peyorativos y autocontrol) del instrumento empleado (Alcázar-Olán et al., 2014a) fue válida para la muestra estudiada de adolescentes mexicanos, lo cual impidió rechazar la hipótesis de investigación. Los pensamientos de enojo son el constructo determinante para sentir frecuentemente esta emoción (Owen, 2011; Wilkowski \& Robinson, 2010). Por lo tanto, el elemento central para esta discusión será el de la estructura de los pensamientos de enojo.

A través de los estudios, no parece haber una estructura definitiva de pensamientos de enojo. El elemento que en más ocasiones ha sido replicado, al igual que en el presente trabajo, es el de ideas de venganza (Alcázar-Olán et al., 2014a; Deffenbacher et al., 2003; Snyder et al., 1997; Suchday et al., 2004; Sukhodolsky et al., 2001). Por lo tanto, la venganza parece ser un elemento esencial en la estructura de los pensamientos de enojo. Con base en la importancia de la generalización del conocimiento científico, esto lleva a sugerir que la venganza podría ser un componente universal de los pensamientos de enojo, más allá de las diferencias culturales. En un sentido clínico y práctico, la implicación es que ambos, el enojo y la venganza, deberían 
considerarse para disminuirlos en los tratamientos, por ejemplo, con habilidades de empatía o de perdón (Eaton \& Struthers, 2006), y así aumentar la efectividad en programas de intervenciones con personas que se enojan fácilmente, o incluso en el tratamiento de personas con tendencia a la violencia física o verbal, entre otras.

Los pensamientos peyorativos del presente estudio han surgido en algunas investigaciones (Deffenbacher et al., 2003; Martin \& Dahlen, 2007; Snyder et al., 1997), pero no en otras (Suchday et al., 2004; Sukhodolsky et al., 2001). Por lo tanto, los pensamientos peyorativos deben considerarse con cierta reserva, debido a que cuentan hasta ahora con un menor grado de generalización. Se requiere de más estudios para evaluar si este tipo de pensamientos se encuentran en otros países.

Con respecto a los pensamientos de control del enojo, estos han sido un componente de estudios previos (Deffenbacher et al., 2003; Suchday et al., 2004). Sin embargo, en dichos estudios y en los presentes resultados, los pensamientos de control del enojo han estado poco asociados, es decir, han tenido bajas correlaciones, con la exacerbación del enojo. Esto tiene dos implicaciones para la teoría y la práctica. Primero, los pensamientos de control y los pensamientos de exacerbación del enojo podrían reflejar procesos cognitivos diferentes, lo cual requeriría, por lo tanto, modos distintos de análisis o estudio. Segundo, los clínicos deberían considerar para el tratamiento no a uno solo, sino a ambos tipos de pensamientos. El fundamento, reflejado en las bajas correlaciones mencionadas, es que el aumento en los pensamientos de control no necesariamente lleva a la disminución de pensamientos iracundos.

En cuanto a diferencias por sexo y en apego a la hipótesis, esta investigación encontró mínimas diferencias, igual que estudios previos (Alcázar-Olán et al., 2014a; Archer, 2004; Martin \& Dahlen, 2007). Las mujeres tuvieron más pensamientos peyorativos que los hombres, aunque la varianza explicada fue pequeña (1.4\%). No se encontraron diferencias en pensamientos de venganza ni en pensamientos de control del enojo. Hombres y mujeres parecen más similares que distintos en sus cogniciones asociadas al enojo. Una implicación de este resultado es que la escala puede ser usada con ambos sexos.

Deben mencionarse algunas limitaciones y sugerencias de investigación. Primero, aunque el estudio partió de constructos válidos en una muestra mexicana de universitarios (Alcázar-Olán et al., 2014a), el hecho de optar por 
dichos constructos llevó a eliminar, automáticamente, constructos distintos que quizá sean relevantes en adolescentes. Por ejemplo, no se estudiaron constructos como exigencias rígidas (esto es, los "deberías"), generalizaciones (por ej., "nunca me comprendes") o atribuciones erróneas (por ej., "tomar algo como personal"). Segundo, en congruencia con el punto anterior, aunque se validaron tres factores, eso no implica que sean los "verdaderos" constructos de los pensamientos de enojo en adolescentes. Queda abierta la posibilidad de probar otros modelos, con otros constructos. Tercero, falta replicar el presente estudio con otras muestras para examinar si los factores encontrados son estables (validación cruzada). De igual forma, deberá analizarse la estabilidad temporal en un estudio test-retest para considerar el uso de la escala en la evaluación de intervenciones. Cuarto, puede criticarse que el presente estudio no incluyó una muestra clínica. Si bien es cierto que no se incluyeron adolescentes que fueron derivados a servicios psicológicos o psiquiátricos por problemas de enojo o agresión, es un hecho que los adolescentes con problemas de enojo muchas veces están "entremezclados" en poblaciones más grandes (Alcázar-Olán \& Deffenbacher, 2013). De no ser así, entonces no habría problemas de agresión o pleitos en las escuelas (Gamboa et al., 2016). Además, esta limitación se superó, al menos parcialmente, en que la Escala de Pensamientos de Enojo discriminó de forma correcta y en la dirección esperada a personas de alto y bajo puntaje en escalas existentes. Además, la división por cuartiles se ha convertido en un estándar en la detección de personas con enojo problemático (DiGiuseppe \& Tafrate, 2003).

En síntesis, la Escala de Pensamientos de Enojo para adolescentes mexicanos demostró validez de constructo, reflejado en que diferenció a personas de alto y bajo puntaje en variables de enojo, agresión y control del enojo. La subescala de venganza fue la que tuvo mayor poder discriminativo al contrastar grupos, seguida por la de pensamientos peyorativos y pensamientos de autocontrol.

Agradecimientos: Los autores agradecemos a las autoridades escolares y a los padres de familia por su apoyo en la realización del presente estudio.

Financiamiento: El presente estudio fue posible con el financiamiento de la Universidad Iberoamericana Puebla (México) para hacer investigación.

\section{REFERENCIAS}

Alcázar-Olán, R. J. \& Deffenbacher, J. L. (2013). High trait anger Mexican youth: characteristics, parental anger, and counseling needs. The Spanish Journal of Psychology, 16, 1-10. https://doi.org/10.1017/sjp.2013.89 
Alcázar-Olán, R. J., Deffenbacher, J. L., Hernández-Guzmán, L. \& de la Chaussée, M. E. (2014a). The angry thoughts scale: Initial development in a Mexican sample. International Journal of Cognitive Therapy, 7, 373-388. http://dx.doi.org/10.1521/ ijct.2014.7.4.373

Alcázar-Olán, R. J., Deffenbacher, J. L., Pool, W., J., \& Reyes, V. (2014b). Developing a valid version of the ML-STAXI to measure anger in Mexican adolescents. In M. G. Penrod \& S. N. Paulk (Eds.), Psychology of Anger (pp. 89-105). Nueva York: Nova Science Publishers.

Alcázar-Olán, R. J., Jurado, S. \& Reyes, V. (2015). El enojo como rasgo de personalidad y agresión física en adolescentes de preparatoria. Psicumex, 5, 89-101. Disponible en https://psicumex.unison.mx/index.php/psicumex/article/view/31

Archer, J. (2004). Sex differences in aggression in real-world settings: A meta-analytic review. Review of General Psychology, 8, 291-322. http://dx.doi. org/10.1037/1089-2680.8.4.291

Ato, M., López, J. J. \& Benavente, A. (2013). Un sistema de clasificación de los diseños de investigación en psicología. Anales de Psicología, 29, 1038-1059. http://dx.doi. org/10.6018/analesps.29.3.178511

Bentler, P. M. (1990). Comparative fit indexes in structural models. Psychological Bulletin, 107, 238-246. http://dx.doi.org/10.1037/0033-2909.107.2.238

Bentler, P. M. (1995). EQS structural equations program manual. Encino, CA: Multivariate Software.

Berkowitz, L. \& Harmon-Jones, E. (2004). More thoughts about anger determinants. Emotion, 4, 151-155. http://dx.doi.org/10.1037/1528-3542.4.2.151

Blake, C. S. \& Hamrin, V. (2007). Current approaches to the assessment and management of anger and aggression in youth: A review. Journal of Child and Adolescent Psychiatric Nursing, 20, 209-221. http://dx.doi.org/10.1111/j.17446171.2007.00102.x

Blanchard-Fields, F. \& Coats, A. H. (2008). The experience of anger and sadness in everyday problems impacts age differences in emotion regulation. Developmental Psychology, 44, 1547-1556. http://dx.doi.org/10.1037/a0013915

Brotman, M.A., Kircanski, K. \& Leibenluft, E. (2017). Irritability in children and adolescents. Annual Review of Clinical Psychology, 13, 317-341. http://dx.doi. org/10.1146/annurev-clinpsy-032816-044941

Cassiello-Robbins, C. \& Barlow, D. H. (2016). Anger: The unrecognized emotion in emotional disorders. Clinical Psychology: Science and Practice, 23, 66-85. http:// dx.doi.org/10.1111/cpsp.12139

Cohen, J. (1988). Statistical power analysis for the behavioral sciences (2nd Ed.). [Version Adobe Reader] http://www.utstat.toronto.edu/ brunner/ oldclass/378f16/readings/CohenPower.pdf

Cohen, R. J., \& Swerdlik, M. E. (2009). Psychological testing and assessment: An introduction to tests and measurement (7th Ed.). Estados Unidos: McGraw-Hill.

Dahlen, E. R. \& Martin, R. C. (2006). Refining the anger consequences questionnaire. Personality and Individual Differences, 41, 1021-1031. http://dx.doi. org/10.1016/j.paid.2006.04.008

Deffenbacher, J. L., Oetting, E. R., Lynch, R. S. \& Morris, C. D. (1996). The expression of anger and its consequences. Behavior Research and Therapy, 34, 575-590. http:// dx.doi.org/10.1016/0005-7967(96)00018-6

Deffenbacher, J. L., Petrilli, R. T., Lynch, R. S., Oetting, E. R. \& Swaim, R. C. (2003). The driver's angry thoughts questionnaire: A measure of angry cognitions 
when driving. Cognitive Therapy and Research, 27, 383-402. http://dx.doi. org/10.1023/A:1025403712897

DiGiuseppe, R. \& Tafrate, R.C. (2003). Anger treatment for adults: A meta-analytic review. Clinical Psychology: Science and Practice, 10, 70-84. http://dx.doi. org/10.1093/clipsy/10.1.70

Eaton, J. \& Struthers, C. W. (2006). The reduction of psychological aggression across varied interpersonal contexts through repentance and forgiveness. Aggressive Behavior, 32, 195-206. http://dx.doi.org/10.1002/ab.20119

Fernandez, E., \& Johnson, S. L. (2016). Anger in psychological disorders: Prevalence, presentation, etiology and prognostic implications. Clinical Psychology Review, 46, 124-135. http://dx.doi.org/10.1016/j.cpr.2016.04.012

Gamboa, A. A., Ortiz, J. A. \& Muñoz, P. A. (2016). Violencia en contextos escolares: percepción de docentes sobre manifestaciones de violencia en instituciones educativas en Cúcuta-Norte de Santander. Psicogente, 20, 89-98. http://doi. org/10.17081/psico.20.37.2420

Hu, L. \& Bentler, P. (1999). Cutoff criteria for fit indexes in covariance structure analysis: conventional criteria versus new alternatives. Structural Equation Modeling, 6, 1-55. https://doi.org/10.1080/10705519909540118

INEGI (2016). Principales causas de mortalidad por residencia habitual, grupos de edad y sexo del fallecido. Recuperado en: http://www.inegi.org.mx/est/contenidos/proyectos/registros/vitales/mortalidad/tabulados/pc.asp?t=14\&c=11817

Martin, R. C. \& Dahlen, E. R. (2007). The angry cognitions scale: A new inventory for assessing cognitions in anger. Journal of Rational-Emotive \& Cognitive-Behavior Therapy, 25, 155-173. https://doi.org/10.1007/s10942-006-0033-2

Messick, S. (1995). Validity of psychological assessment. American Psychologist, 50, 741-749. http://dx.doi.org/10.1037/0003-066X.50.9.741

Moscoso, M. (2000). Estructura factorial del inventario multicultural latinoamericano de la expresión de la cólera y la hostilidad. Revista Latinoamericana de Psicología, 32(2), 321-343. Disponible en http://www.redalyc.org/pdf/805/80532204.pdf

Nunnally, J. C. \& Bernstein, I. H. (1995). Teoría psicométrica (3a ed.). México: McGraw Hill.

Owen, J. M. (2011). Transdiagnostic cognitive processes in high trait anger. Clinical Psychology Review, 31, 193-202. https://doi.org/10.1016/j.cpr.2010.10.003

Phillips, L. H., Henry, J. D., Hosie, J. A., \& Milne, A. B. (2006). Age, anger regulation and well-being. Aging \& Mental Health, 10, 250-256. https://doi. org/10.1080/13607860500310385

Potegal, M., Stemmler, G., \& Spielberger, C. D. (2010). International handbook of anger: Constituent and concomitant biological, psychological, and social processes. Nueva York: Springer.

Satorra, A. \& Bentler, P. M. (2001). A scaled difference chi-square test statistic for moment structure analysis. Psychometrika, 66, 507-514. https://doi.org/10.1007/ BF02296192

Smith, C. A., Haynes, K. H., Lazarus, R. S. \& Pope, L. K. (1993). In search of the "hot" cognitions: Attributions, appraisals, and their relation to emotion. Journal of Personality and Social Psychology, 65, 916-929. https://doi.org/10.1023/A:1019876601693

Snyder, C. R., Crowson, J. J., Houston, B. K., Kurylo, M. \& Poirier, J. (1997). Assessing hostile automatic thoughts: Development and validation of the HAT scale. Cognitive Therapy and Research, 21, 477-492. https://doi.org/10.1023/A:1021988511695

Spielberger, C.D. (1999). Professional manual for the State-Trait Anger Expression Inventory-2 (STAXI-2). Odessa, FL: Psychological Assessment Resources. 
Spielberger, C. D., \& Reheiser. E. C. (2009). Assessment of emotions: Anxiety, anger, depression, and curiosity. Applied Psychology: Health and Well-being, 1, 271-302. https://doi.org/10.1111/j.1758-0854.2009.01017.x

Suchday, S., Carter, M. M., Ewart, C. K., Larkin, K., T. \& Desiderato, O. (2004). Anger cognitions and cardiovascular recovery following provocation. Journal of Behavioral Medicine, 27, 319-341. http://dx.doi.org/10.1023/B:JOBM.0000042408.80551. e1

Sukhodolsky, D. G., Golub, A., \& Cromwell, E. N. (2001). Development and validation of the anger rumination scale. Personality and Individual Differences, 31, 689-700. https://doi.org/10.1016/S0191-8869(00)00171-9

Takebe, M., Takahashi, F., \& Sato, H. (2016). Anger rumination as a risk factor for trait anger and anger-in: A longitudinal study. Personality and Individual Differences, 101, 451-455. http://dx.doi.org/10.1016/j.paid.2016.06.038

Van de Vijver, F.J.R., \& Poortinga, Y. H. (2005). Conceptual and methodological issues in adapting tests. In R. K. Hambleton, P. F. Merenda, \& C. D. Spielberger (Eds.), adapting educational and psychological tests for cross-cultural assessment (pp. 39-63). New Jersey: Lawrence Erlbaum Associates.

Wilkowski, B. M. \& Robinson, M. D. (2010). The anatomy of anger: an integrative cognitive model of trait anger and reactive aggression. Journal of Personality, 78, 9-38. https://doi.org/10.1111/j.1467-6494.2009.00607.x

World Health Organization (2018). Adolescent health epidemiology, leading causes of death. Retrieved from: http://www.who.int/maternal_child_adolescent/epidemiology/adolescence/en/

Zimprich, D. \& Mascherek, A. (2012). Measurement invariance and age-related differences of trait anger across the adult lifespan. Personality and Individual Differences, 52, 334-339. https://doi.org/10.1016/j.paid.2011.10.030

\section{(cc) $\mathbf{B Y}$}


